\title{
Molecular docking, MM/GBSA and 3D-QSAR studies on EGFR inhibitors
}

\author{
RAJU BATHINI, SREE KANTH SIVAN, SABIHA FATIMA and VIJJULATHA MANGA* \\ Molecular Modeling and Medicinal Chemistry Group, Department of Chemistry, University College of \\ Science, Osmania University, Hyderabad 500 007, India \\ e-mail: vijjulathamanga@gmail.com
}

MS received 13 November 2015; revised 2 May 2016; accepted 4 May 2016

\begin{abstract}
Epidermal growth factor receptor (EGFR) is the first growth factor receptor proposed as a target for cancer therapy. Molecular modeling protocols like molecular docking, molecular mechanics/generalized born surface area (MM/GBSA) calculations and three dimensional-quantitative structure activity relationship (3D-QSAR) studies were performed on 45 molecules to understand the structural requirements for EGFR tyrosine kinase inhibitors. Conformation for all the molecules obtained from molecular docking were used as is for 3D-QSAR analysis. Comparative molecular field analysis (CoMFA) and comparative molecular similarity indices analysis (CoMSIA) models were obtained by performing partial least square analysis on 35 training molecules and these models were validated using 10 test moleucles. The models showed good statistical results in terms of $r^{2}, q_{\text {loo }}^{2}$ and $r_{\text {pred }}^{2}$ values. Information rendered from 3D-QSAR model and sitemap analysis was used to optimize lead molecule to design prospective inhibitors. Improvement in EGFR binding affinity can be achieved by substitutional modification on phenyl ring attached to alkynyl group with bulkier hydrogen bond donor and acceptor substituents that can increase favourable interaction with the receptor.
\end{abstract}

Keywords. Epidermal growth factor receptor (EGFR); Extra precision (XP) docking; 3D-QSAR; Comparative molecular field analysis (CoMFA); Comparative molecular similarity indices analysis (CoMSIA); Molecular mechanics; generalized born surface area (GBSA).

\section{Introduction}

Epidermal growth factor receptor (EGFR) is a transmembrane glycoprotein consisting of a single polypeptide chain of 1186 amino acids. ${ }^{1}$ It belongs to a family of receptor tyrosine kinases (TKs) that includes EGFR, ErbB-2, ErbB-3 and ErbB-4 (also known as HER-1, HER-2, HER-3, and HER-4). ${ }^{2}$ The EGFR and its family members are composed of an extracellular ligand-binding domain, a transmembrane domain, and an intracellular domain. When epidermal growth factor (EGF), binds to the ligand-binding domain, the EGF receptor forms a homodimer or heterodimer with other members of EGFR family. This activates the intrinsic kinase domain that in turn triggers autophosphorylation events on specific tyrosine residues within the cytoplasmic tail activity. ${ }^{3,4}$ Downstream single proteins then initiate several signal transduction cascades, leading to DNA synthesis, and cell proliferation. ${ }^{5}$ EGFR is a crucial regulator in several cell phenotypes, such as cell migration, adhesion, proliferation, and immune response in the human skin making it a key target for anti-tumor strategy.

\footnotetext{
*For correspondence
}

Early studies on EGFR inhibition have reported many compounds having inhibitory activity, these included 4-anilinoquinazoline derivatives, ${ }^{6-8}$ arylaminopyrimidines $^{9,10}$ and pyrrole triazines derivatives. ${ }^{11,12}$ Lapatinib, which is a potent dual EGFR/ErbB2 inhibitor, approved by FDA for the treatment of breast cancer (figure 1), is a modified 4-Anilinoquinazoline derivative. ${ }^{13}$ Recently, pyrimidine derivatives like anilinopyrimidines, thienopyrimidines, and alkenyl-methylpyrimidine were reported as EGFR inhibitors. ${ }^{14-18}$ All these skeletons possess a pyrimidine ring portion that bind with EGFR. The crystal structures of EGFR bound to inhibitors have two hydrogen bond interactions specifically with the active site residues Met793 and Asp800. Met793 played a key role in the inhibitory activity of EGFR. In an ongoing effort, we report receptor based 3D-QSAR studies using comparative molecular field analysis (CoMFA) ${ }^{19,20}$ and comparative molecular similarity indices analysis $(\text { CoMSIA })^{21}$ methodology on EGFR inhibitors. Partial least square (PLS) ${ }^{22}$ based statistical analysis was carried out on 45 molecules to identify the correlation. Contour maps generated enabled us to explain the observed variation in activity and guided us to design new molecules. 


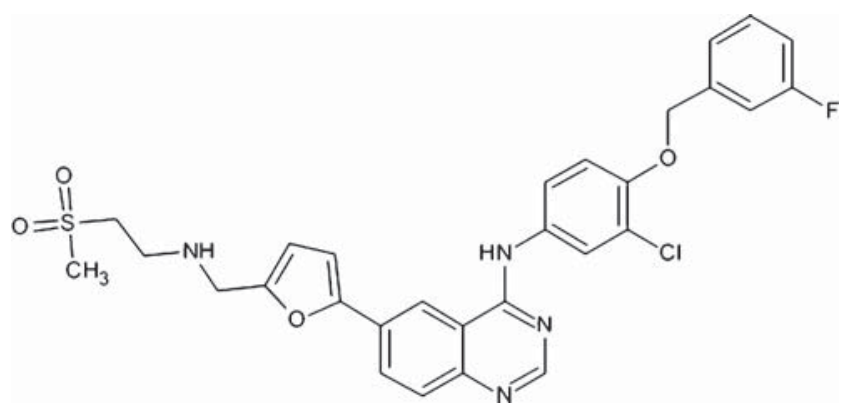

Figure 1. Lapatinib - Dual EGFR/ErbB-2 inhibitor.

\section{Methodology}

\subsection{Ligand preparation}

All molecular modeling calculations were performed on a Linux operating system. A total of $45^{14-18}$ molecules were available with reported $\mathrm{IC}_{50}$ values for inhibition of EGFR tyrosine kinase. The kinase inhibition assay $\mathrm{IC}_{50}$ determinations were measured with the homogeneous time resolved fluorescence (HTRF) KinEASE-TK assay. Three dimensional structures of these inhibitors were sketched using Mastreo build panel in Schrödinger 2010 suite, and the $\mathrm{IC}_{50}$ values were converted to $\mathrm{pIC}_{50}$ values $\left(\mathrm{pIC}_{50}=-\log \mathrm{IC}_{50}\right)($ table 1$)$.

The ligprep module of Schrodinger was used to generate all possible states at a physiological $\mathrm{pH}$ range $7 \pm 2$ and produces lowest potential energy conformer of the ligand using an OPLS_2005 force field. All these ligands post preparation was used for molecular docking studies and ADME properties using Qikprop module of Schrodinger Suite.

\subsection{Protein preparation}

Crystal structure of EGFR tyrosine kinase bound with lapatinib with resolution of $2.4 \AA$ was downloaded from RCSB protein data bank.(http://www.rcsb.org/ $\mathrm{pdb} /$ ) (PDB id 1xkk). Protein was prepared using protein preparation wizard module of Schrodinger Suite. All the water molecules and heteroatoms except lapatinib (FMM) were deleted and hydrogen atoms were added to the crystal structure. The complex was submitted to minimization to relieve steric clashes using the OPLS 2005 force field. Sitemap module was used to characterise features of binding sites. Hydrophobic and hydrophilic maps are generated which are dispersed as donor, acceptor, and hydrophobic regions.

\subsection{Molecular docking}

Active site for docking ligands was defined using Receptor Grid Generation module in Glide 5.6. ${ }^{23}$
Co-crystallied ligand was selected and grid was generated around the active site of EGFR intrinsic domain, with a receptor van der Waals scaling for non-polar atoms as $0.9 .^{24,25}$ Molecules selected from literature (figure 2) were prepared by LigPrep application. These molecules were docked into the grid generated from EGFR protein structure. Two different docking protocols, standard precision (SP) and extra precision (XP) were employed to analyze binding modes of the known inhibitors from the literature.

\subsection{Prime MM/GBSA calculations}

To estimate relative binding affinities of protein ligand complexes, Molecular mechanics with generalized born surface area (MM/GBSA) is employed. The relative binding free energy $\Delta G_{\text {bind }}$ was estimated according to the following equation:

$$
\begin{aligned}
\Delta \mathrm{G}_{\text {bind }}= & \left.\mathrm{E}_{\text {complex }} \text { (minimized }\right) \\
& -\left[\mathrm{E}_{\text {ligand }} \text { (unbound, minimized }\right) \\
& \left.\left.+\mathrm{E}_{\text {receptor }} \text { (unbound, minimized }\right)\right]
\end{aligned}
$$

Where $\Delta \mathrm{G}_{\text {bind }}$ is the calculated relative free energy that includes both ligand and receptor strain energy. $\mathrm{E}_{\text {complex }}$ (minimized) is the MM/GBSA energy of the minimized complex, and $\mathrm{E}_{\text {ligand }}$ (unbound, minimized) is the MM/GBSA energy of the ligand after removing it from the complex and allowing it to relax. $\mathrm{E}_{\text {receptor }}$ (unbound, minimized) is the MM/GBSA energy of protein after separating it from the ligand. ${ }^{26,27}$ Prime MM/GMSA modules was used for calculating the relative binding free energy for each molecule. The protein ligand complexes obtained from XP docking were subjected to MM/GBSA calculations.

\subsection{CoMFA and CoMSIA studies}

CoMFA and CoMSIA studies were carried out as described by Carmer et al., ${ }^{19,20}$ and Fatima et al. ${ }^{28}$ The process is briefly described here. Dock pose of each ligand that showed two hydrogen bond interactions with the receptor were imported into SYBYLX-2.1 molecular modeling program package ${ }^{29}$ and GasteigerHuckel $^{30}$ charges were assigned. Standard Tripos force fields were employed for CoMFA and CoMSIA analysis. A 3D cubic lattice of dimension $4 \AA$ in each direction with each lattice intersection of a regularly spaced grid of $2.0 \AA$ was created. Steric and electrostatic parameters were calculated in CoMFA fields while hydrophobic, acceptor and donor parameters in addition to steric and electrostatic were calculated in CoMSIA fields at each lattice. The $\mathrm{sp}^{3}$ carbon atom was used as a 
Table 1. Experimental and predicted activity of EGFR tyrosine kinase inhibitors along with Dock score (Kcal/mol) and $\Delta \mathrm{G}_{\mathrm{bind}}(\mathrm{Kcal} / \mathrm{mol})$.

\begin{tabular}{|c|c|c|c|c|c|c|c|}
\hline Mol & $\mathrm{IC}_{50(\mu \mathrm{M})}$ & $\mathrm{pIC}_{50}$ & $\begin{array}{l}\text { Predicted pIC } 50 \\
\text { CoMFA }\end{array}$ & $\begin{array}{l}\text { Predicted pIC } \\
\text { CoMSIA }\end{array}$ & $\begin{array}{l}\text { SP Dock Score } \\
(\mathrm{Kcal} / \mathrm{mol})\end{array}$ & $\begin{array}{l}\text { XP Dock Score } \\
\text { (Kcal/mol) }\end{array}$ & $\begin{array}{c}\Delta \mathrm{G}_{\mathrm{bind}} \\
(\mathrm{Kcal} / \mathrm{mol})\end{array}$ \\
\hline 1 & 0.650 & 6.190 & 6.050 & 6.120 & -11.260 & -11.326 & -121.746 \\
\hline 2 & 0.022 & 7.660 & 8.040 & 7.910 & -12.700 & -12.128 & -132.827 \\
\hline 3 & 0.003 & 8.520 & 7.490 & 8.240 & -11.740 & -12.197 & -133.303 \\
\hline 4 & 0.009 & 8.050 & 7.720 & 7.820 & -11.500 & -11.572 & -135.183 \\
\hline 5 & 0.190 & 6.720 & 7.000 & 7.470 & -11.940 & -12.147 & -125.868 \\
\hline 6 & 0.079 & 7.100 & 7.370 & 7.380 & -11.700 & -11.527 & -140.538 \\
\hline $7 *$ & 0.030 & 7.520 & 7.416 & 7.648 & -11.440 & -11.427 & -143.336 \\
\hline 8 & 0.055 & 7.260 & 7.120 & 7.220 & -12.130 & -12.309 & -128.936 \\
\hline $9 *$ & 0.094 & 7.030 & 6.750 & 6.970 & -11.020 & -12.369 & -136.369 \\
\hline $10 *$ & 0.920 & 6.040 & 5.920 & 5.700 & -9.620 & -9.702 & -90.464 \\
\hline $11^{*}$ & 1.100 & 5.960 & 5.789 & 5.903 & -9.930 & -10.085 & -100.213 \\
\hline 12 & 47.000 & 4.330 & 4.670 & 4.490 & -9.520 & -9.527 & -96.164 \\
\hline 13 & 22.000 & 4.660 & 5.110 & 5.000 & -9.090 & -8.096 & -109.857 \\
\hline 14 & 10.000 & 5.000 & 5.920 & 5.460 & -10.310 & -10.175 & -108.267 \\
\hline $15^{*}$ & 0.068 & 7.170 & 6.708 & 7.113 & -10.810 & -11.282 & -125.437 \\
\hline 16 & 0.065 & 7.190 & 7.190 & 7.230 & -11.160 & -11.902 & -115.438 \\
\hline 17 & 0.590 & 6.230 & 7.060 & 6.450 & -11.460 & -11.119 & -117.256 \\
\hline 18 & 0.034 & 7.470 & 7.190 & 7.140 & -11.020 & -11.571 & -143.399 \\
\hline 19 & 0.240 & 6.620 & 7.290 & 7.040 & -11.700 & -11.636 & -135.572 \\
\hline 20 & 0.028 & 7.550 & 7.320 & 7.480 & -11.580 & -12.491 & -140.553 \\
\hline $21^{*}$ & 0.018 & 7.740 & 6.971 & 6.808 & -11.720 & -12.462 & -128.446 \\
\hline 22 & 0.014 & 6.940 & 6.960 & 6.840 & -11.430 & -12.010 & -132.853 \\
\hline 23 & 0.003 & 8.520 & 8.110 & 8.280 & -11.480 & -11.976 & -128.161 \\
\hline 24 & 0.019 & 7.720 & 7.430 & 7.880 & -11.070 & -11.846 & -130.904 \\
\hline 25 & 0.156 & 6.810 & 7.210 & 7.160 & -11.050 & -12.239 & -131.088 \\
\hline 26 & 0.013 & 7.890 & 7.350 & 7.080 & -10.930 & -11.846 & -127.077 \\
\hline $27 *$ & 4.130 & 5.380 & 6.371 & 6.682 & -11.210 & -11.451 & -128.013 \\
\hline 28 & 0.038 & 7.420 & 7.270 & 7.370 & -11.380 & -11.690 & -127.590 \\
\hline 29 & 0.011 & 7.960 & 7.740 & 7.360 & -10.620 & -11.284 & -139.209 \\
\hline $30 *$ & 0.009 & 8.040 & 8.279 & 8.235 & -11.740 & -11.722 & -137.283 \\
\hline 31 & 9.700 & 5.010 & 4.700 & 4.990 & -9.540 & -9.584 & -108.649 \\
\hline 32 & 5.600 & 5.250 & 5.050 & 5.480 & -9.360 & -8.639 & -89.460 \\
\hline 33 & 72.400 & 4.140 & 3.980 & 3.810 & -9.020 & -7.540 & -84.807 \\
\hline 34 & 3.500 & 5.460 & 4.980 & 5.710 & -9.020 & -8.215 & -93.839 \\
\hline 35 & 26.600 & 4.580 & 4.230 & 3.990 & -10.800 & -10.727 & -100.470 \\
\hline 36 & 8.600 & 5.070 & 5.410 & 5.410 & -8.620 & -7.755 & -102.330 \\
\hline 37 & 0.009 & 8.050 & 8.160 & 8.220 & -13.310 & -13.321 & -137.448 \\
\hline $38 *$ & 0.080 & 7.100 & 6.113 & 6.429 & -11.410 & -9.700 & -138.488 \\
\hline 39 & 0.180 & 6.740 & 6.720 & 6.530 & -11.550 & -12.447 & $-129 . .403$ \\
\hline 40 & 0.108 & 6.970 & 6.630 & 6.600 & -10.800 & -11.434 & -115.789 \\
\hline 41 & 0.129 & 6.890 & 7.010 & 6.840 & -11.290 & -11.592 & -129.656 \\
\hline $42 *$ & 0.048 & 7.320 & 6.626 & 6.551 & -10.620 & -11.936 & -112.935 \\
\hline 43 & 0.627 & 6.200 & 6.610 & 6.180 & -11.430 & -12.536 & -124.355 \\
\hline 44 & 0.821 & 6.090 & 6.670 & 6.190 & -11.190 & -12.043 & -125.053 \\
\hline 45 & 2.138 & 6.140 & 5.660 & 6.030 & -9.830 & -11.587 & -107.014 \\
\hline
\end{tabular}

* represents test set molecules.

probe atom to generate steric (Lennard-Jones potential) field energies and a charge of +1 to generate electrostatic (Coulombic potential) field energies. A distance dependent dielectric constant of 1.00 was used. Steric and electrostatic fields were condensed to +30.00 $\mathrm{Kcal} / \mathrm{mol}$.

PLS analysis was performed to correlate computed fields with biological activity $\left(\mathrm{pIC}_{50}=-\log \mathrm{IC}_{50}\right)$ for the data set of ligands. Molecules were randomly divided into training and test set of 35 and 10, respectively, as 3:1 ratio. The $\mathrm{pIC}_{50}$ difference between most active and least active molecule in the dataset is 4.38 $\log$ units. The training set was used to generate the QSAR model and test set was used to validate the generated model. In the PLS statistical analysis, biological activity values of ligands were used as dependent 
variables. To improve the signal-to-noise ratio column filtering value (s) was set to $2.0 \mathrm{Kcal} / \mathrm{mol}$ by omitting lattice points whose energy variations were below the threshold. Leave-one-out (LOO) cross-validations were performed to determine the optimum number of components (ONC) and coefficient $\mathrm{q}_{\mathrm{loo}}^{2}$. The obtained ONC was applied to derive the final QSAR model. Validation of CoMFA and CoMSIA derived model was performed by predicting the activity of test set compounds.

\section{Results and Discussion}

The accuracy of molecular conformation for Quantitative Structure Activity Relationship (QSAR) studies
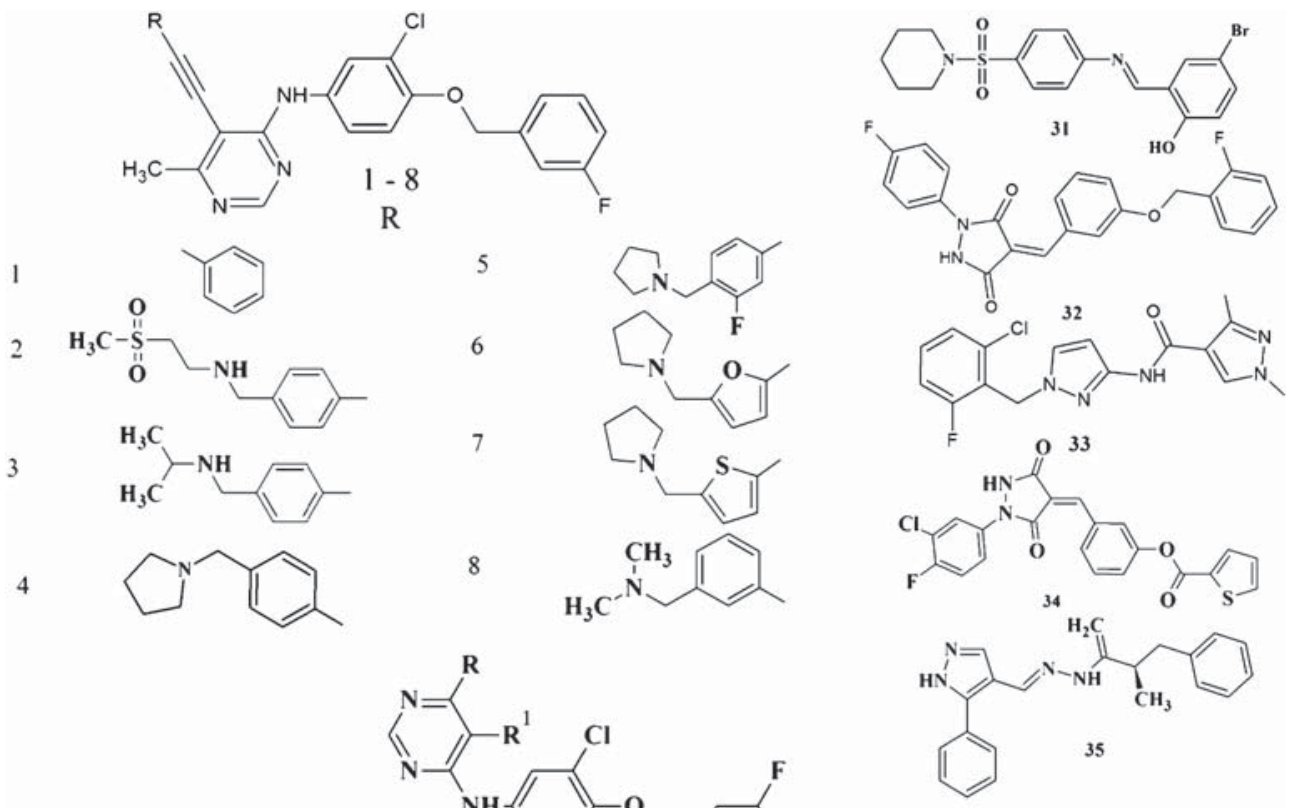

$\mathrm{R}$

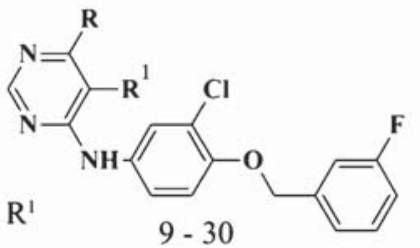

$\mathrm{R}$

$\mathrm{R}^{1}$

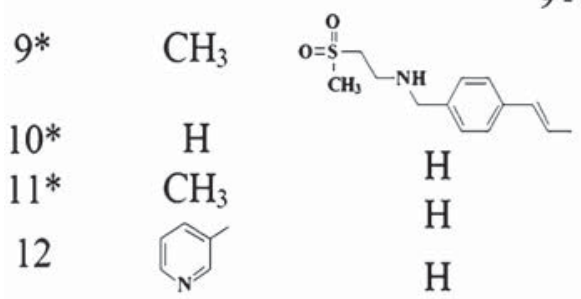

13

14<smiles>C1=CC2=CC=CC2=C1</smiles>

H

$\mathrm{H}$

$15^{*} \quad \mathrm{CH}_{3}$

$16 \mathrm{CH}_{3}$

$17 \quad \mathrm{CH}_{3}$

$18 \quad \mathrm{CH}_{3}$

$19 \mathrm{CH}_{3}$
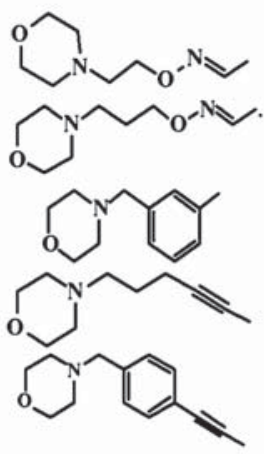

20

$\mathrm{CH}_{3}$

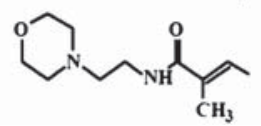

$21^{*}$

22

23

$\mathrm{CH}_{3}$<smiles>CC=C(C)C(=O)NCCCC1CCOCC1</smiles>

$\mathrm{CH}_{3}$<smiles>C/C=C/C(=O)NCCN1CCOCC1</smiles>

24

$\mathrm{CH}_{3}$<smiles>CC=C(F)C(=O)NCCC(C)(C)O</smiles>

25

26

$27^{*}$

$\mathrm{CH}_{3}$<smiles>CCCCCC(C)C(C)C(C)CC</smiles>

28

$\mathrm{CH}_{3}$

29

$\mathrm{CH}_{3}$

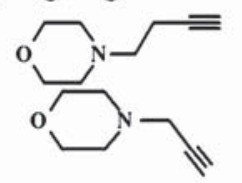

30 *

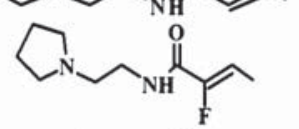<smiles>C=CC=NOCCN1CCOCC1</smiles>

$\mathrm{CH}_{3}$

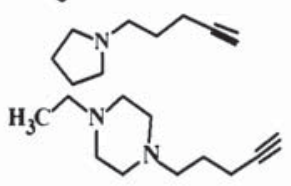

Figure 2. Structures of EGFR Inhibitors. *indicates test molecules. 
<smiles>Cc1ccc(N2NC(=O)/C(=C\c3ccc(OCc4ccccc4)cc3)C2=O)cc1Cl</smiles>

37

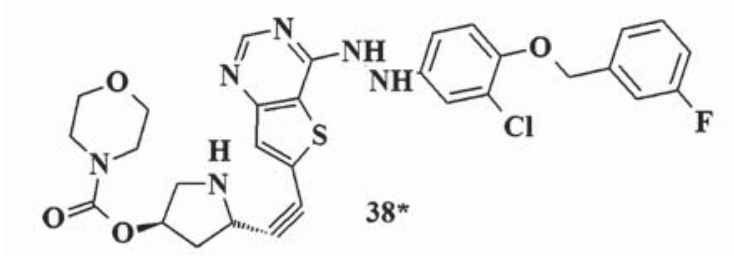<smiles>[R]c1cccc(Oc2cc(Nc3ccc(OCc4cccc(F)c4)c(Cl)c3)ncn2)c1</smiles>

40<smiles>[R]NC(C)=O</smiles>

41

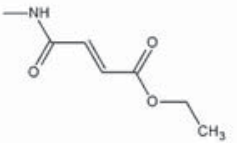

$42^{*}$<smiles>CNC(=O)CC#N</smiles>

43<smiles>CNC(=O)Cc1cc(F)c(F)cc1F</smiles>

44

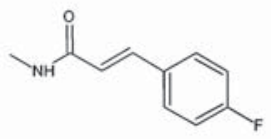

45

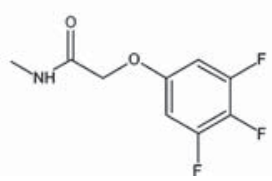

Figure 2. (Continued).

is a most important criteria, and the most favorable bioactive conformer selection is a tough task. Molecular docking of active compounds into the active site of the protein is one of the methods used for obtaining the active conformation. These molecules were docked into the active site (lapatinib binding site) of EGFR to obtain the receptor based conformations (figure 2). The RMSD between the predicted conformation and the observed X-ray Crystallographic conformation of the compound (Lapatinib) equaled $1.92 \AA$, a value that suggests the reliability of docking protocol in producing the experimentally observed binding mode for EGFR inhibitors. Dock poses analysis of all the molecules showed similar hydrogen bond interactions with active site residues. Molecules were having specific hydrogen bond interactions between $\mathrm{N} 1$ of the pyrimidine ring and amine in the tail region with hinge region amino acid Met793 and Asp800, respectively. These hydrogen bond interactions were also observed for the co-crystalized ligand (lapatinib), and it also showed a halogen interaction with gate keeper amino acid Thr790. DFG binding motif is also a crucial part for inhibitor binding to EGFR, lapatinib and most of the pyrimidine derivative showed a hydrophobic $(\pi-\pi)$ interaction with Phe856.

Molecular mechanics/generalized born surface area (MM/GBSA) calculations were performed for best ranking molecules in XP mode. MM/GBSA calculations were performed to estimate relative binding affinity of ligands to the receptor. In principle, MM/GBSA is used for free energy based ranking of ligands belonging to a congeneric series. MM/GBSA and docking protocols employed in the present work for assessing ligand affinities to EGFR allow protein flexibility and therefore, give more reliable results.

The list of inhibitors along with their $\mathrm{pIC}_{50}$, dock scores from SP, XP and $\Delta \mathrm{G}_{\text {bind }}$ values from Prime have been tabulated in table 1. Dock score (XP dock score) vs. Experimental $\mathrm{pIC}_{50}$ and $\Delta \mathrm{G}_{\text {bind }}$ vs. Experimental $\mathrm{pIC}_{50}$ gave a correlation coefficient value (r) of 0.728 and 0.782 , respectively, which shows a significant 
relation between biological activity vs. docking and free energy calculations. The high correlation depicts the accuracy of the docking protocol. The correlation of $\mathrm{MM} / \mathrm{GBSA} \mathrm{G}_{\mathrm{bind}}$ values and experimental values is in acceptable range of $78 \%\left(r=0.78\right.$ and $\left.r^{2}=0.61\right)$. Even though a few molecules showed higher MM/GBSA values than best active molecule 3 and 23. The variation in binding affinity with respect to experimental activity can be correlated to lesser aqueous solubility of these molecules (ADME properties in supplementary data). Molecule 37 (lapatinib) is the co-crystallized ligand in the protein structure used for docking studies (pdb id $1 \mathrm{xxk}$ ) hence its docking and binding values are higher compared to the best active molecule, other molecules had bulkier hydrophobic group (heterocyclic saturated groups like morpholine, pyrrolidine) that showed favourable interaction with hydrophobic amino acids residues (Leu 718, Cys 797, Leu 1001 and Met 1002) and also an electrostatic interaction with Asp 800. Scatter plot of $\mathrm{pIC}_{50}$ versus $\mathrm{XP}$ dock score and $\Delta \mathrm{G}_{\mathrm{bind}}$ values are shown in figure 3. Usually in 3D QSAR, all the molecules in the dataset are superimposed onto the best active molecule. Instead, the most promising poses (best GLIDE Score along with most physiologically similar positions) of each molecule has been considered for 3D QSAR analysis. Overlay of dock pose of each ligand is shown in figure 4.

CoMFA and CoMSIA analysis were carried out on these reported inhibitors. The molecules were divided into a training set consisting of 35 molecules for the derivation of QSAR model and a test set of 10 molecules for validation of derived model. CoMFA and CoMSIA statistical analysis are summarized in table 2. Statistical data shows $\mathrm{q}_{\mathrm{loo}}^{2}$ of 0.638 and $0.672, \mathrm{r}^{2} 0.88$ and 0.929 , respectively, for CoMFA and CoMSIA. The $\mathrm{q}_{\text {loo }}^{2}$ and $\mathrm{r}^{2}$ values indicate a good internal predictive ability of this model. The SEE value of 0.448 and 0.345 , respectively, for CoMFA and CoMSIA indicate error in prediction of activity from the obtained model, as the model is derived from a training set of 35 molecules the

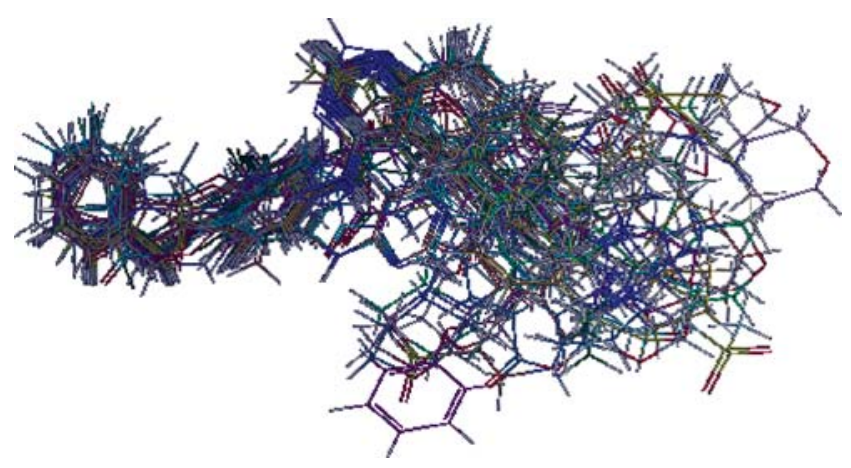

Figure 4. Dock pose alignment of EGFR inhibitors.

Table 2. PLS results in summary.

\begin{tabular}{lcc}
\hline Statistical parameters & CoMFA & CoMSIA \\
\hline Training set & 35 & 35 \\
Test set & 10 & 10 \\
$\mathrm{q}_{\text {loo }}^{2}$ & 0.638 & 0.672 \\
ONC & 3 & 3 \\
SEE & 0.448 & 0.345 \\
$\mathrm{r}^{2}$ & 0.880 & 0.929 \\
$\mathrm{~F}_{\text {ration }}$ & 75.645 & 134.713 \\
$\mathrm{r}_{\text {pred }}^{2}$ & 0.530 & 0.520 \\
& Fraction of field contributions & \\
Steric & 0.619 & 0.124 \\
Electrostatic & 0.381 & 0.219 \\
Hydrophobic & - & 0.178 \\
Donor & - & 0.214 \\
Acceptor & - & 0.265 \\
\hline F & & \\
\hline
\end{tabular}

$\overline{\mathrm{F}_{\text {ratio }}}=$ Fisher test value; $\mathrm{q}_{\text {loo }}^{2}=$ cross-validated correlation coefficient by leave one out method; $\mathrm{r}^{2}=$ conventional correlation coefficient; $r_{\text {pred }}^{2}=$ cross-validated correlation coefficient on test set; ONC, optimum number of components; SEE, standard error of estimate.
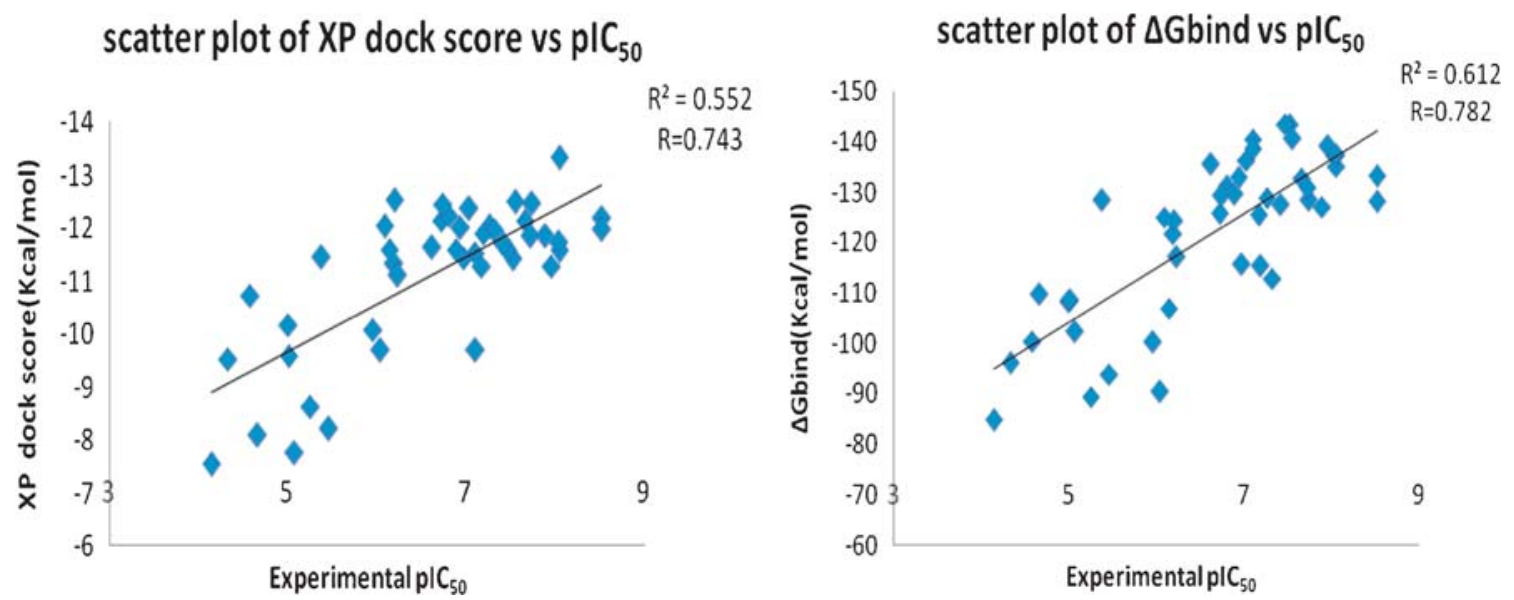

Figure 3. Scatter plot of $\mathrm{pIC}_{50} v s$. XP dock score and $\Delta \mathrm{G}_{\text {bind }}$ values. 
value seem to be optimum with respect to the $r^{2}$ values obtained for the models, the error estimate would have been less for a larger set. Test set molecules excluded from the model generation were used to predictive ability of the model. Predictive correlation coefficient $r_{\text {pred }}^{2}$ 0.53 for CoMFA and 0.52 for CoMSIA, respectively, wich indicates good external predictive ability of the model.

Scatter plots for actual and predicted $\mathrm{pIC}_{50}$ values for training and test sets of CoMFA and CoMSIA studies are shown in figure 5. Observed and predictive $\mathrm{pIC}_{50}$ values of these molecules are provided in table 1.

CoMFA and CoMSIA contour maps were generated to visualize information content of the 3D QSAR models. Modifications required to design new molecules are suggested by molecular fields (contour maps) that define favorable or unfavorable interaction energies of aligned molecules. Contour maps of CoMFA denote the region in the space where molecules would favorably or unfavorably interact with the receptor while CoMSIA contour maps symbolize specified regions where the presence of a particular group with certain physicochemical property binds to the receptor. CoMFA and CoMSIA results were graphically interpreted by field contribution maps using "STDEVCOEFF" field type. All contours represented default 80 and 20\% level contribution for favored and unfavored regions. ${ }^{19,20}$ Molecule 3, the most potent inhibitor within the series was incorporated on the map to aid visualization. Variation in activity is discussed based on the contour maps analyzes the characterstic features of binding sites.

Steric contour maps of CoMFA and CoMSIA models are similar as seen in figures $6 \mathrm{a}$ and $6 \mathrm{~b}$, respectively; the green and yellow areas represent favored and disfavored regions, respectively. Large green contour is seen near the substituted benzylamine ring, suggesting substitution with a bulky group at this position will increase the activity. The most active molecule (3) shows substituted benzylamine ring incorporated into the green region. A yellow contour is seen around the pyrimdine ring, suggesting more bulky substituents in this area will significantly decrease the biological activity. Figure 7
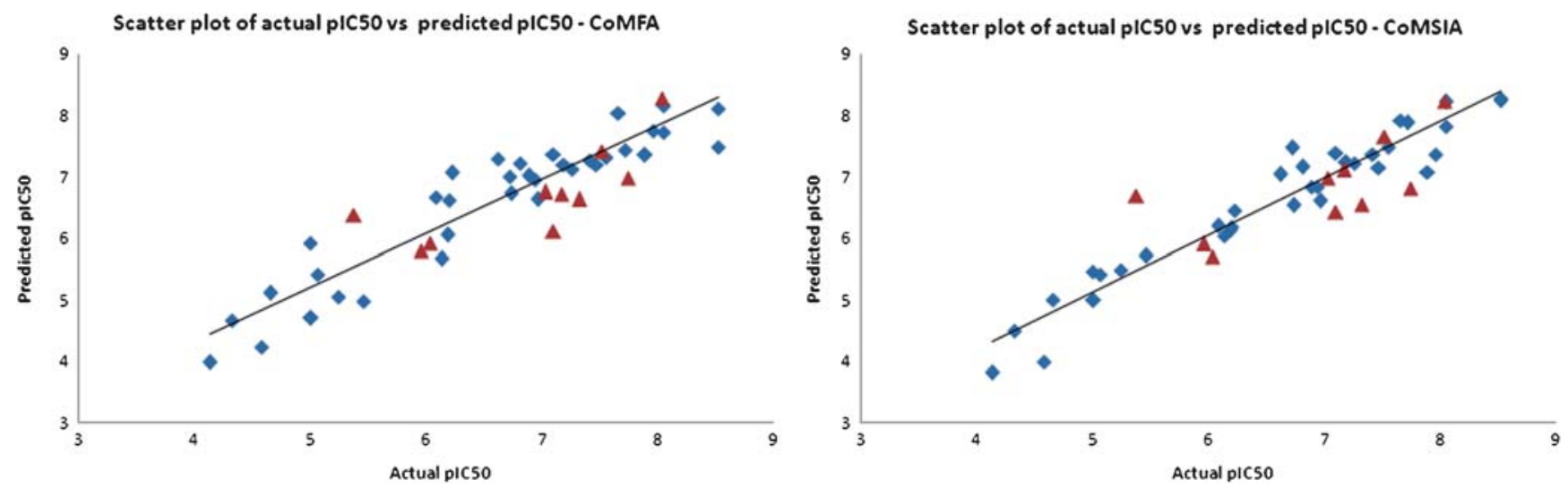

Figure 5. Scatter plot for the actual and predicted $\mathrm{pIC}_{50}$ values, the training set is represented in squares and test set in triangles.
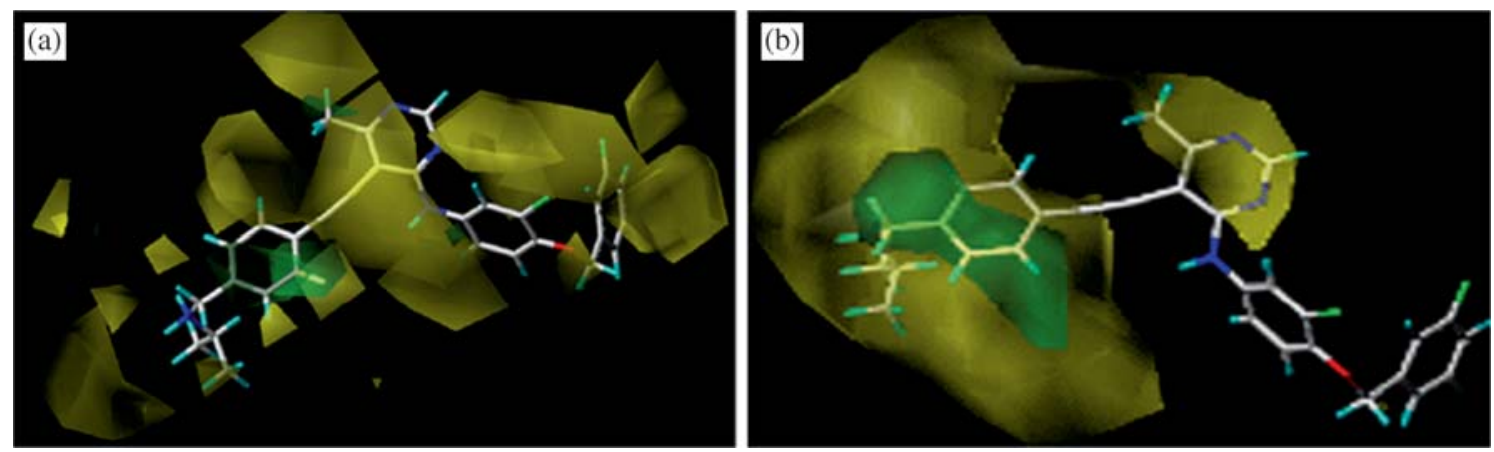

Figure 6. CoMFA and CoMSIA steric fields. (a) CoMFA and (b) CoMSIA standard deviation (SD* coefficient) contour map illustrating steric features in combination with molecule 3. Green contours represent favorable bulky group substitution at that point while yellow regions are disfavorable for activity. 
shows electrostatic contour maps where the red contour represents electronegative charge favoring and blue contour represents the electropositive charge favoring, respectively; blue contour on the side chain of substituted benzylamine ring indicates lesser electronegative substituents may increase the activity. Red contour on benzyloxy phenyl ring suggests that high electronegative substituents like flourine at this position are essential for biological activity as in the case of the most active molecule 3.

Hydrophobic contour are embodied in figure 8a, yellow and white areas represent hydrophobic and hydrophilic preferred regions. Three small yellow contours are seen, one at phenyl ring, one at methyl substituent on pyrimidine ring and third on benzyloxy attached phenyl ring. Bulkier hydrophobic group substitution at this position may increase the activity of the molecules since this region is also steric favored. A white contour is observed at the phenyl ring suggesting hydrophilic groups in this region will increase activity.

Acceptor and donor contour maps are shown in figure $8 \mathrm{~b}$, magenta and cyan fields indicate the hydrogen bond acceptor and donor favored regions whereas red and purple fields imply disfavored regions. In molecule 3 , a donor disfavored purple contour is seen in the vicinity of the pyrimidine ring. Magenta contours are away from the molecule, and a small cyan contour is observed near the NH of the side chain phenyl ring. Site map analysis of the receptor showed similar hydrophobic, hydrogen bond donor and hydrogen bond acceptor regions as obtained from the CoMSIA analysis. These analyses of contour maps and sitemap assisted us to identify structural requirements for receptor binding and inhibition. New molecules have been designed based on these structural requirements as shown in figure 9. The dock pose of molecule 3 shown in figure 10a shows yellow hydrophobic region covering the phenyl rings that has
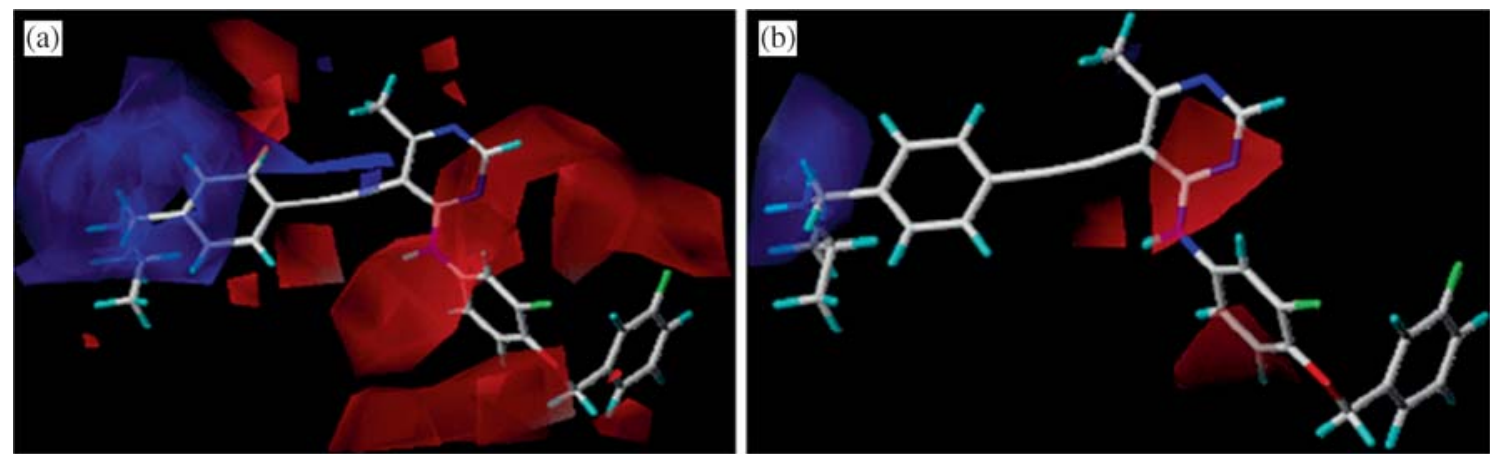

Figure 7. CoMFA and CoMSIA electrostatic fields. (a) CoMFA and (b) CoMSIA electrostatic standard deviation (SD* coefficient) contour maps illustrating electrostatic features in combination with molecule 3. Red contours indicate negative charge favoring activity, whereas blue contours indicate positive charge favoring activity.
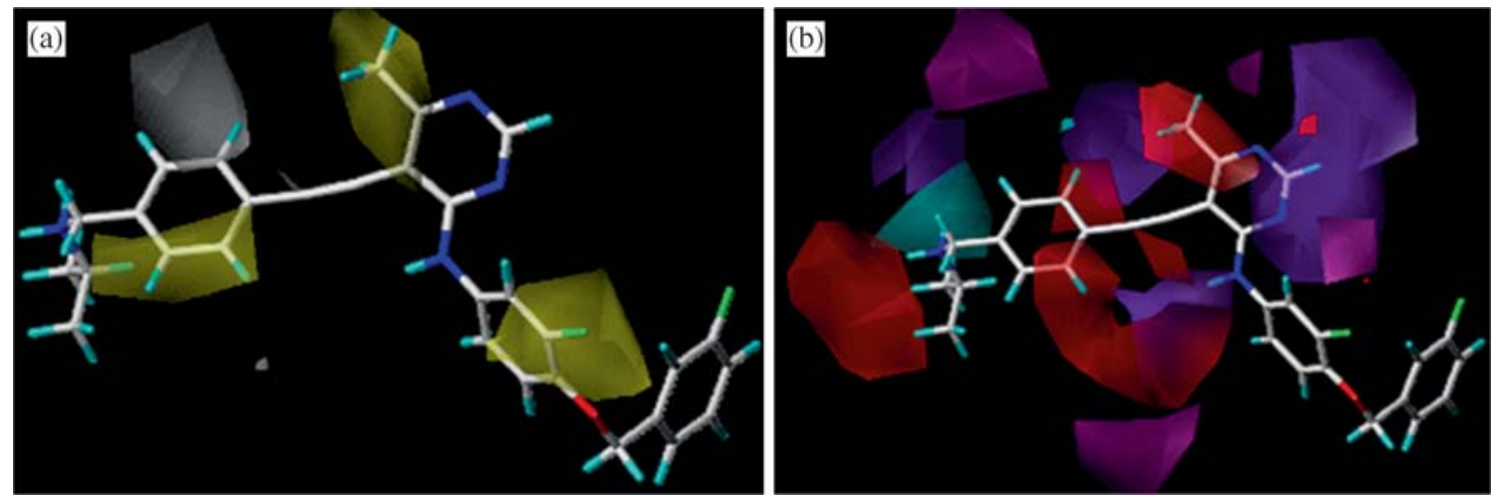

Figure 8. CoMSIA SD* coefficient contour maps illustrating, (A) hydrophobic, and (B) donor and acceptor features in combination with molecule 3. Yellow contour represents the hydrophobic favored region, white indicates the hydrophilic favored regions. The purple contour represents H-bond donor disfavored regions while cyan indicates $\mathrm{H}$-bond donor favored region. Magenta and red contour represent H-bond acceptor favored and disfavored regions, respectively. 


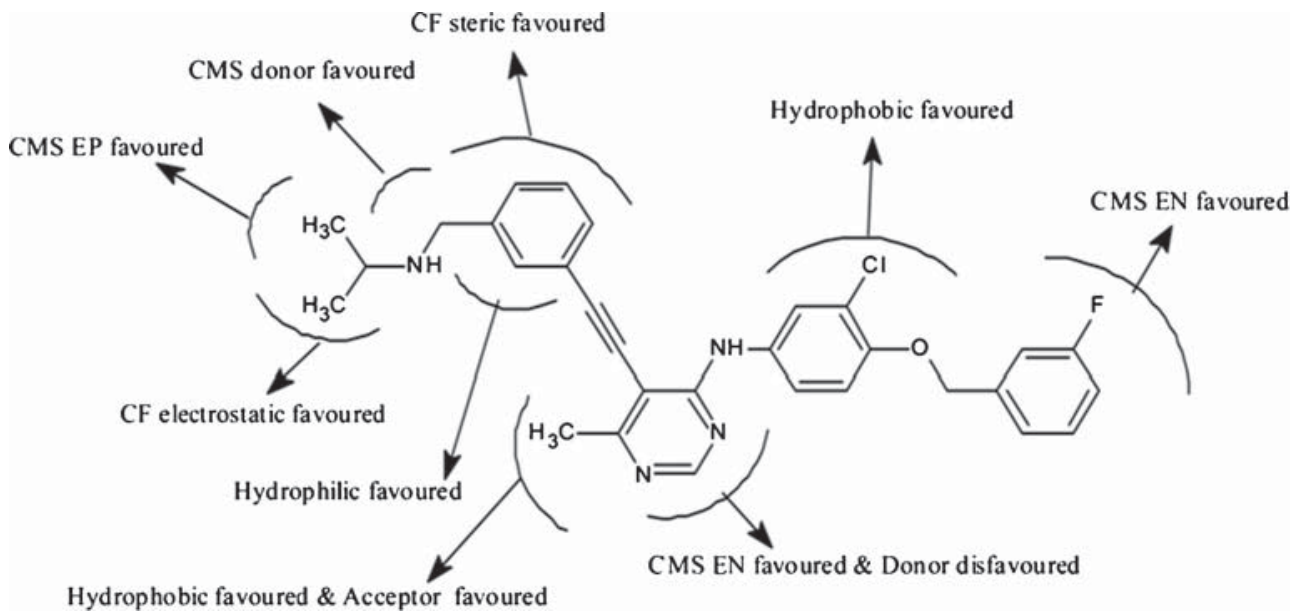

Figure 9. Structural requirements for EGFR inhibitors obtained from CoMFA (CF) and CoMSIA (CMS) contour map and sitemap analysis.
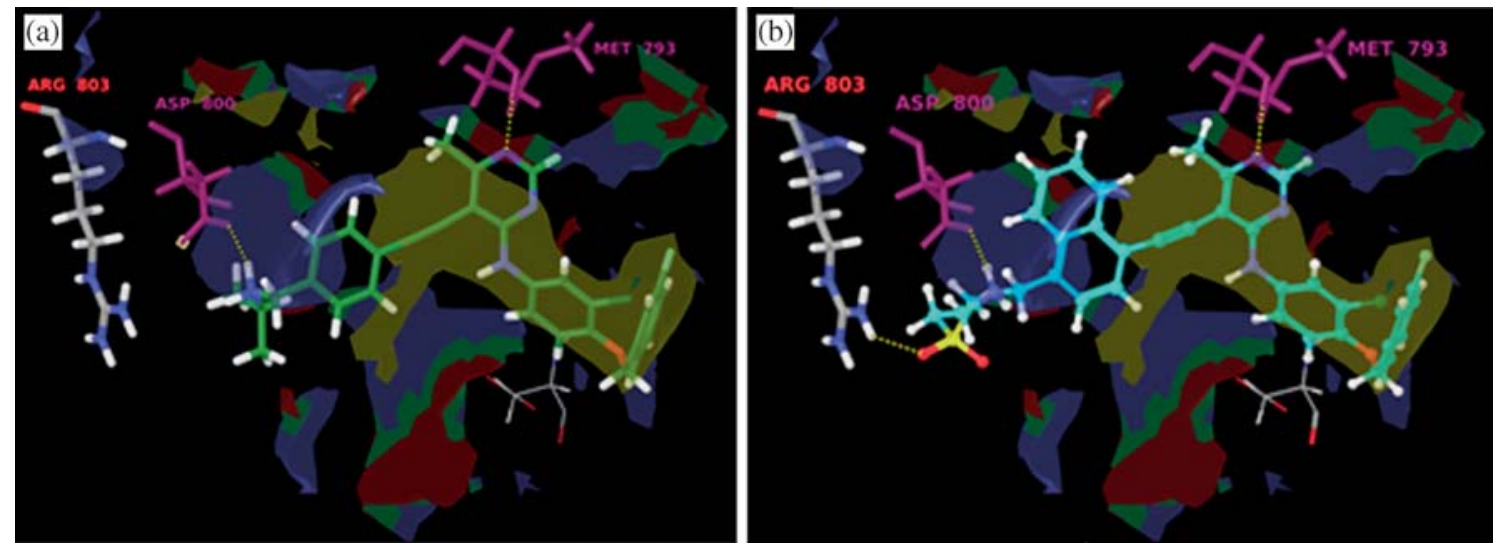

Figure 10. Sitemap analysis of, (a) most active molecule 3 of the data set, (b) dock pose of newly designed molecule N4 showing increased number of hydrogen bond interaction with active site amino acids. Yellow region represents hydrophobic, blue represents hydrogen bond donor, red represents hydrogen bond acceptor, and green represents hydrophilic.

$\pi-\pi$ interaction with Phe856, a hydrogen bond acceptor red region is seen over the nitrogen of pyrimidine group that interacts with Met793. Blue hydrogen bond donor region is seen near amine group of the tail region having salt bridge interaction with Asp800.

Bulky groups with hydrophilic character were substituted on the phenyl ring attached to alkynyl group. Groups like sulfonyl and amine were substituted to the side chain of phenyl ring resulting in more hydrogen bond interaction between the receptor and ligand (figure 10b). Designed molecules were docked into the active site, and they showed similar interactions with comparable dock score and predicted activity with respect to the the most active compound 3 . Figure 10 shows dock poses of the most potent molecule 3 and newly designed molecule N4. These molecules are embedded in the protein along with hydrogen bond interaction with active site amino acids. N4 showed one extra hydrogen bond interaction with Arg803. Methylsulphonyl side chain attached to the amine allows it to occupy the cavity having increased hydrogen bond interactions. Predicted $\mathrm{pIC}_{50}$ values were calculated and found to be better. Their structures and predicted $\mathrm{pIC}_{50}$ are given in table 3. ADME properties of the newly designed molecules were calculated and found to be in range as that of pyrimidine derivatives used in the present study. The toxicity properties of these molecules were calculated using pKCSM online server. ${ }^{31}$ The new molecules showed similar properties as data set molecules, namely AMES toxicity, CYP and hERG inhibition, etc. The ADMET properties have been calculated and provided as Supplementary Information for both the new molecules and data set molecules. 
Table 3. Structures of newly designed molecules with predicted $\mathrm{pIC}_{50}$ values, Dock score $(\mathrm{Kcal} / \mathrm{mol})$ and $\Delta \mathrm{G}_{\mathrm{bind}}(\mathrm{Kcal} / \mathrm{mol})$.<smiles>Cc1ncnc(Nc2ccc(OCc3cccc(F)c3)c(Cl)c2)c1C#CP</smiles>

\begin{tabular}{|c|c|c|c|c|c|c|}
\hline Mol & $\mathrm{R}$ & $\begin{array}{c}\text { Pred pIC } \\
\text { CoMFA }\end{array}$ & $\begin{array}{l}\text { Pred pIC } \\
\text { CoMSIA }\end{array}$ & $\begin{array}{l}\text { SP Dock score } \\
(\mathrm{Kcal} / \mathrm{mol})\end{array}$ & $\begin{array}{l}\text { XP Dock score } \\
\text { (Kcal/mol) }\end{array}$ & $\begin{array}{c}\Delta \mathrm{G}_{\text {bind }} \\
(\mathrm{Kcal} / \mathrm{mol})\end{array}$ \\
\hline N1 & & 7.855 & 7.387 & -11.801 & -12.166 & -141.336 \\
\hline $\mathrm{N} 2$ & & 7.704 & 8.229 & -12.821 & -13.368 & -126.719 \\
\hline N3 & & 7.884 & 7.595 & -12.453 & -12.889 & -146.801 \\
\hline N4 & & 7.503 & 7.285 & -13.176 & -13.242 & -150.500 \\
\hline N5 & & 7.681 & 7.288 & -12.524 & -13.237 & -144.551 \\
\hline
\end{tabular}

\section{Conclusions}

To understand the binding mode of molecules to receptors and rationalize structural requirements for inhibiton, molecular modeling tools like docking, MM/GBSA and 3D-QSAR studies are helpful. In the present study, molecular docking (SP and XP) and prime MM/GBSA calculations were performed to understand the binding mode of inhibitors with EGFR. CoMFA and CoMSIA methodologies were used to build a model for obtaining structural requirement for EGFR inhibitors. The generated model showed good statistical results in terms of high $\mathrm{r}^{2}$ and $\mathrm{q}^{2}$ values for all the models, this indicates the ablility of QSAR model to predict potent inhibitors from designed molecules. Based on detailed analysis of contour maps and sitemap, it is evident that improvement in EGFR binding affinity can be achieved by substitutional modification on phenyl ring attached to alkynyl group with bulky hydrophilic substituents. Maintaining hydrophobic character at phenyloxy group attached to pyrimidine ring of the best active molecule 3 has enhanced the binding affinity.

\section{Supplementary Information (SI)}

ADMET properties of newely designed molecules, Tables and Figures of Superimposition of structure pose and docked pose of co-crystalized ligand with RMS deviation of $1.92 \AA$, have been incorporated in SI, available at www.ias.ac.in/chemsci. 


\section{Acknowledgements}

We gratefully acknowledge support for this research from the University Grants Commission (UGC-UPE, No. 23/UGC/UPE/FAR/OU/2014), New Delhi, India, Council of Scientific and Industrial Research, Department of Science and Technology, New Delhi, India. $\mathrm{RB}$ would like to acknowledge financial support from CSIR for a research fellowship. We wish to express our gratitude to Dept of Chemistry, Osmania University for providing facilities to carry out the research work. We also acknowledge Schrödinger Inc. for GLIDE software, Tripos Inc. for SYBYLX-1.2.

\section{References}

1. Ciardiello F and Tortora G 2008 N. Engl. J. Med. 358 1160

2. Pao W and Chmielecki J 2010 Nat. Rev. Cancer 10760

3. Citri A and Yarden Y 2006 Nat. Rev. Mol. Cell. Biol. 75

4. Downward J, Yarden Y, Mayes E, Scrace G, Totty N, Stockwell P, Ullrich A, Schlessinger J and Waterfield M D 1984 Nature 307521

5. Oda K, Matsuoka Y, Funahashi A and Kitano H 2005 Mol. Syst. Biol. 1200

6. Smaill J B, Palmer B D, Rewcastle G W, Denny W A, McNamara D J, Dobrusin E M, Bridges A J, Zhou H, Showalter H D H, Winters R T, Leopold W R, Fry D W, Nelson J M, Slintak V, Elliot W L, Roberts B J, Vincent P W and Patmore S J 1999 J. Med. Chem. 421803

7. Tsou H-R, Mamuya N, Johnson B D, Reich M F, Gruber B C, Ye F, Nilakantan R, Shen R, Discafani C, DeBlanc R, Davis R, Koehn F E, Greenberger L M, Wang Y-F and Wissner A 2001 J. Med. Chem. 442719

8. Wissner A, Berger D M, Boschelli D H, Floyd M B, Greenberger L M, Gruber B C, Johnson B D, Mamuya N, Nilakantan R, Reich M F, Shen R, Tsou H-R, Upeslacis E, Wang Y F, Wu B, Ye F and Zhang N 2000 J. Med. Chem. 433244

9. Xu G, Abad M C, Connolly P J, Neeper M P, Struble G T, Springer B A, Emanuel S L, Pandey N, Gruninger R H, Adams M, Moreno-Mazza S, Fuentes-Pesquera A R and Middleton S A 2008 Bioorg. Med. Chem. Lett. 184615

10. Xu G, Searle L L, Hughes T V, Beck A K, Connolly P J, Abad M C, Neeper M P, Struble G T, Springer B A, Emanuel S L, Gruninger R H, Pandey N, Adams M, Moreno-Mazza S, Fuentes-Pesquera A R, Middleton S A and Greenberger L M 2008 Bioorg. Med. Chem. Lett. 183495

11. Mastalerz H, Chang M, Chen P, Fink B E, Gavai A, Han W-C, Johnson W, Langley D, Lee F Y, Leavitt K,
Marathe P, Norris D, Oppenheimer S, Sleczka B, Tarrant J, Tokarski J S, Vite G D, Vyas D M, Wong H, Wong T W, Zhang $\mathrm{H}$ and Zhang G 2007 Bioorg. Med. Chem. Lett. 174947

12. Mastalerz H, Chang M, Chen P, Fink B E, Gavai A, Han W -C, Johnson W, Langley D, Lee F Y, Leavitt K, Marathe P, Norris D, Oppenheimer S, Sleczka B, Tarrant J, Tokarski J S, Vite G D, Vyas D M, Wong H, Wong T W, Zhang $\mathrm{H}$ and Zhang G 2007 Bioorg. Med. Chem. Lett. 172828

13. Rusnak D W, Lackey K, Affleck K, Wood E R, Alligood K J, Rhodes N, Keith B R, Murray D M, Knight W B, Mullin R J and Gilmer T M 2001 Mol. Cancer Ther. 185

14. Li S, Guo C, Zhao H, Tang Y and Lan M 2012 Bioorg. Med. Chem. 20877

15. Li S, Guo C, Zhao H, Tang Y and Lan M 2012 Bioorg. Med. Chem. 224004

16. Waterson A G, Petrov K G, Hornberger K R, Hubbard R D, Sammond D M, Smith S C, Dickson H D, Caferro T R, Hinkle K W, Stevens K L, Dickerson S H, Rusnak D W, Spehar G M, Wood E R, Griffin R J and Uehling D E 2009 Bioorg. Med. Chem. Lett. 191332

17. Suzuki N, Shiota T, Watanabe F, Haga N, Murashi T, Ohara T, Matsuo K, Omori N, Yari H, Dohi K, Inoue M, Iguchi M, Sentou J and Wada T 2011 Bioorg. Med. Chem. Lett. 211601

18. Suzuki N, Shiota T, Watanabe F, Haga N, Murashi T, Ohara T, Matsuo K, Omori N, Yari H, Dohi K, Inoue M, Iguchi M, Sentou J and Wada T 2012 Bioorg. Med. Chem. Lett. 22456

19. Cramer R D, Patterson D E and Bunce J D 1988 J. Am. Chem. Soc. 1105959

20. Cramer R D, Patterson D E and Bunce J D 1988 Quant. Struct. Act. Relat. 718

21. Klebe G, Abraham U and Mietzner T 1994 J. Med. Chem. 374130

22. Wold S, Johansson A and Cochi M 1993 In 3D QSAR in Drug Design, H Kubinyi (Ed.) (Leiden: ESCOM) p. 523

23. Schrödinger L L C 2010 New York Glide Version 56

24. Friesner R A, Banks J L, Murphy R B, Halgren T A, Klicic J J, Mainz D T, Repasky M P, Knoll E H, Shelley M, Perry J K, Shaw D E, Francis P and Shenkin P S 2004 J. Med. Chem. 471739

25. Taverna D M and Goldstein R A 2002 Proteins 46105

26. Srivastava H K and Sastry G N 2012 J. Chem. Inf. Model 523088

27. Munnaluri R, Sivan S K and Manga V 2015 Med. Chem. Res. 24829

28. Fatima S, Bathini R, Sivan S K and Manga V $2012 \mathrm{~J}$. Recept. Signal Transduction 32214

29. Sybyl-X 2.1 2012 (Tripos Associates: St. Louis, MO)

30. Gasteiger J and Marsili M 1980 Tetrahedron 363219

31. Douglas E V P, Blundell T L and David B A 2015 J. Med. Chem. 584066 